\title{
Design of metal-organic frameworks: from theoretical structure and reactivity predictions to mechanochemical synthesis and screening
}

\author{
$\underline{\text { Yizhi Xu }}{ }^{1}$, James Darby ${ }^{2}$, Joseph Marrett ${ }^{3}$, Hatem Titi ${ }^{3}$, Tomislav Friščić ${ }^{3}$, Andrew Morris ${ }^{4}$, Mihails Arhangelskis ${ }^{1}$
}

${ }^{1}$ Faculty of Chemistry, University of Warsaw, Warsaw 02-093, Poland; ${ }^{2}$ Engineering Department, University of Cambridge, Cambridge CB2 1PZ, U.K; ${ }^{3}$ Department of Chemistry, McGill University, Montreal H3A OB8, Canada; ${ }^{4}$ School of Metallurgy and Materials, University of Birmingham, Edgbaston, Birmingham B15 2TT, U.K.;

$$
\text { y.xu7@student.uw.edu.pl }
$$

Metal-organic frameworks (MOFs) are microporous materials with many exciting applications, such as gas storage and separation, catalysis, platforms for artificial photosynthesis and energetic materials. The wide range of applications is strictly related to the modular node-and-linker composition, where different combinations of building blocks yield materials with various properties. The presence of a vast number of combinations for different node and linker, however, poses a real challenge for the experimental MOF design.

An $a b$ initio crystal structure prediction (CSP) method for MOFs has been reported by our group recently, and the method is based on the $a b$ initio random structure searching (AIRSS) [1] and Wyckoff Alignment of Molecules (WAM) [2] algorithms. In this publication, a wide range of existing MOF structures have been investigated. Herein, we will demonstrate the first examples for the prediction of new MOF materials from metal azolate framework (MAF) and hexafluorosilicate families using our CSP method, combined with experimental mechanochemical synthesis and crystal structure determination. The solvent-free mechanochemical synthesis guided by theoretical structure prediction provides for an efficient and green approach to MOF design.

The concept of MOF design goes beyond just the prediction of crystal structures. The connections between the crystal structures and chemical reactivity of freshly designed MOFs will also be studied by utilizing periodic density functional theory (DFT). Furthermore, our theory-based MOF structure and property predictions will be validated experimentally via mechanochemical screening and thermal studies, and ultimately aiming to improve our understanding of MOFs.

[1] Pickard, C. J.; Needs, R. J. (2011). J. Phys. Condens. Matter 23, 53201.

[2] Darby, J. P.; Arhangelskis, M.; Katsenis, A. D.; Marrett, J. M.; Friščić, T.; Morris, (2020). Chem. Mater. 32, 5835-5844.

\section{Keywords: Metal-organic frameworks, crystal structure prediction}

